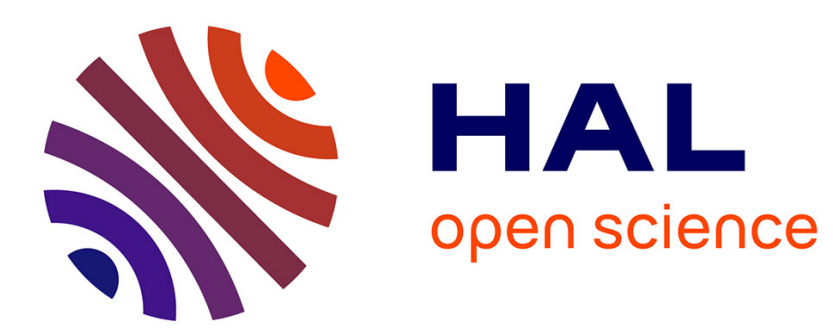

\title{
Les détachements transfrontaliers d'intérimaires du Luxembourg vers la Lorraine
}

Rachid Belkacem, Cathel Kornig, François Michon, Christophe Nosbonne, Benoît Scalvinoni

\section{> To cite this version:}

Rachid Belkacem, Cathel Kornig, François Michon, Christophe Nosbonne, Benoît Scalvinoni. Les détachements transfrontaliers d'intérimaires du Luxembourg vers la Lorraine. La Revue de l'IRES, 2018, 93 (3), pp.51-78. 10.3917/rdli.093.0051 . hal-02284927

\section{HAL Id: hal-02284927 \\ https://hal.science/hal-02284927}

Submitted on 13 Sep 2019

HAL is a multi-disciplinary open access archive for the deposit and dissemination of scientific research documents, whether they are published or not. The documents may come from teaching and research institutions in France or abroad, or from public or private research centers.
L'archive ouverte pluridisciplinaire HAL, est destinée au dépôt et à la diffusion de documents scientifiques de niveau recherche, publiés ou non, émanant des établissements d'enseignement et de recherche français ou étrangers, des laboratoires publics ou privés. 


\title{
Les détachements transfrontaliers d'intérimaires du Luxembourg vers la Lorraine
}

\author{
Rachid BELKACEM, Cathel KORNIG, Francois MICHON, \\ Cbristophe NOSBONNE et Benoît SCALVINONI ${ }^{1}$
}

Les règles européennes concernant le détachement de travailleurs entre Étatsmembres de l'Union européenne sont aujourd'hui de nouveau en débat. L'intérim transfrontalier est l'une des formes assez méconnue de détachement. Cet article s'intéresse aux flux de détachements sur intérim du Luxembourg vers la Lorraine. Le statut luxembourgeois de l'intérim est à plusieurs égards avantageux. II n'est pas rare que des résidents français soient détachés en mission d'intérim en France par des agences luxembourgeoises, parfois non loin de leur domicile. La sédimentation de telles pratiques de part et d'autre des frontières, voire leur banalité, questionne bien sûr les règles nationales ou européennes qui les encadrent.

Dans l'Union européenne (UE), le travail en détachement, c'est la situation d'un travailleur temporairement affecté par son employeur dans un autre État-membre que celui où il travaille habituellement. Ces pratiques de détachement sont encadrées par les règles européennes et limitées à des situations spécifiques (encadré 1). S’ils représentent moins de $1 \%$ de l'emploi total, l'on estime à plus de 2 millions le nombre de travailleurs aujourd'hui détachés dans l'un des pays de l'Union européenne (UE, 2016). Les formes prises par les détachements comme d'ailleurs l'importance ou

1. Rachid Belkacem est enseignant-chercheur au 2L2S, Université de Lorraine : rachid. belkacem@univ-lorraine.fr ; Cathel Kornig est chercheure associée à Aix Marseille Université, CNRS, Lest, Aix-en-Provence : cathel.kornig@icloud.com ; Christophe Nosbonne est enseignantchercheur au 2L2S, Université de Lorraine : christophe.nosbonne@univ-lorraine.fr François Michon est directeur de recherches émérite CNRS : frmichon@mac.com ; Benoît Scalvinoni est docteur en sociologie : benoit.scalvinoni@gmail.com. Ce travail provient d'une étude réalisée pour Force ouvrière dans le cadre de l'Agence d'objectifs de l'Ires (Belkacem, Kornig, Michon, Montcharmont, Nosbonne, Scalvinoni, 2016) disponible sur : https:// goo.gl/ATXCZD. 


\section{Quelques repères règlementaires sur les détachements de travailleurs au sein de l'UE}

Comme l'a rappelé Antoine Math (2006), la libéralisation des services, à la fois dans le cadre de l'accord général sur le commerce des services (AGCS) et dans celui de l'espace économique européen (EEE) a favorisé le développement des détachements de travailleurs. Les normes encadrant les prestations de service à l'international n'ont que peu évolué depuis la directive 96/71/CE de 1996.

Trois types de détachement entrent dans le champ d'application de cette directive : le détachement classique, le détachement intra-groupe et les missions d'intérim dans une entreprise localisée dans un autre pays membre. Elle fixe un noyau dur de dispositions ${ }^{1}$ qui s'imposent aux entreprises procédant à des détachements de travailleurs (art. 3.1). Il s'agit des périodes maximales de travail et des périodes minimales de repos ; la durée minimale des congés annuels payés ; les taux de salaire minimum ; la sécurité, la santé et l'hygiène au travail ; les mesures protectrices applicables aux conditions de travail et d'emploi des femmes, des jeunes et des enfants, et notamment des femmes enceintes ; et, enfin, les conditions de détachement des travailleurs intérimaires.

Le règlement $(C E) n^{\circ} 883 / 2004{ }^{2}$ sur la coordination des systèmes de Sécurité sociale des États-membres précise le cadre réglementaire des détachements. II prévoit en effet pour le travailleur détaché le maintien au régime de Sécurité sociale de l'État-membre d'origine. Pour cela, le détachement ne doit pas excéder 24 mois et le travailleur ne peut être envoyé pour remplacer un travailleur parvenu au terme de son détachement. D'une manière générale, le salarié détaché (ou son employeur) doit adresser un formulaire A1 aux organismes de l'inspection du travail du pays d'accueil afin d'attester du maintien au régime du pays d'envoi. Préalablement à son détachement, le travailleur doit avoir été affilié pendant une durée minimale d'un mois au régime de Sécurité sociale du pays d'envoi. De plus, un délai de carence de deux mois doit séparer deux détachements.

La directive d'exécution du 15 mai 2014 complète la directive 96/71/CE. Avec elle, les entreprises donneuses d'ordre sont tenues pour responsables des fraudes commises par leurs sous-traitants. Les États-membres s'engagent à assurer le contrôle des entreprises concernées. La France a transposé dans sa législation la responsabilité conjointe et solidaire du donneur d'ordre. Trois lois (juillet 2014, août 2015 dite « loi Macron », août 2016 dite « loi El Khomri ») précisent le sens de cette responsabilité conjointe et solidaire :

- obligation de vigilance du donneur d'ordre. II doit vérifier que les entreprises auxquelles il a recours ont satisfait à leurs différents engagements déclaratifs en matière de détachement de travailleurs ; que les sous-traitants répondent bien aux demandes éventuelles des inspecteurs du travail ;

- engagement de leur responsabilité financière qui les oblige à se substituer à un sous-traitant défaillant, par exemple en cas de non-paiement des salaires 
et charges dues aux travailleurs détachés ou encore en cas d'hébergement indigne de ces travailleurs.

Deux réunions des 28 ministres européens du Travail et des Affaires sociales se sont tenues au Luxembourg, respectivement le 15 juin et le 23 octobre 2017. L'accord européen du 23 octobre 2017 est un compromis entre les États-membres. II traite de l'égalité des rémunérations entre travailleurs détachés et travailleurs locaux pour un même poste et sur un même site. Il réduit à 12 mois (au lieu de 24) la durée maximale des détachements.

1. Ces dispositions ont été transposées en France au sein de l'article L. 1262-4 du Code du travail.

2. Voir le règlement (CEE) $n^{\circ} 1408 / 1971$ ainsi que son règlement d'application (CEE) $n^{\circ} 574 / 72$, remplacés le $1^{\text {er }}$ mai 2010 par le règlement $(C E) n^{\circ} 883 / 2004$, lui-même modifié par le règlement (CE) $n^{\circ} 988 / 2009$ et son règlement d'application (CE) $n^{\circ} 987 / 2009$.

le sens des flux de travailleurs ne sont pas toujours ceux que l'on croit. La France se positionne ainsi comme le troisième pays d'envoi des travailleurs détachés et le second pays d'accueil.

Selon les données de la Direction générale du travail française, les détachements de travailleurs sont concentrés sur les territoires frontaliers. Près de $45 \%$ des déclarations enregistrées par les services de l'État le sont dans des départements situés aux abords des frontières, parmi lesquels figurent les départements lorrains de Moselle et de Meurthe-et-Moselle (DGT, 2016). 21271 travailleurs détachés ont été recensés dans cette région en 2015, soit 7,4 \% des 286000 travailleurs détachés en France cette même année. Dans ce contexte, une partie des travailleurs dits «frontaliers $»^{2}$ sont en détachement professionnel et en particulier en détachement d'intérimaires. Ces détachements d'intérimaires entre le Luxembourg et la Lorraine font l'objet de cet article. On le verra, cette déclinaison atypique du détachement rompt assez radicalement avec les images classiquement véhiculées sur les travailleurs détachés.

Nous rappellerons tout d'abord (I) les principales dimensions socioéconomiques de ces pratiques de détachement, au sein de la Grande Région Sarre-Lorraine-Luxembourg (Saar-Lor-Lux) qui regroupe autour du Luxembourg deux Länder allemands (la Sarre et la Rhénanie Palatinat), une région belge (la Wallonie) et une partie de la région française GrandEst correspondant à l'ancienne région Lorraine, et où le détachement transfrontalier sur mission d'intérim s'est développé et normalisé. Les agences de travail temporaire implantées au Luxembourg jouent un rôle

2. Dans I'UE, on entend par travailleurs frontaliers les personnes qui habitent et qui travaillent dans deux États-membres différents, traversant ainsi quotidiennement (ou au moins une fois par semaine) une ou plusieurs frontière(s) nationale(s) pour se rendre sur leur lieu de travail. 
déterminant entre Luxembourg et Lorraine, en allant par exemple recruter des travailleurs intérimaires en Lorraine avant de les envoyer en mission auprès d'une entreprise utilisatrice située... en Lorraine.

Nous montrerons ensuite (II) comment, d'un pays à l'autre, les différentiels de dynamiques économiques et de législations sociale et fiscale ont contribué au développement des mobilités transfrontalières (et parmi elles, les détachements de travailleurs). Elles ont autorisé le développement d'intermédiaires de l'emploi au Luxembourg (les agences d'intérim), qui ont pu se spécialiser dans l'activité de détachement de travailleurs au-delà des frontières luxembourgeoises vers la Lorraine (et ailleurs).

Nous essaierons enfin de comprendre les éléments actuels du débat en reproduisant ce que peuvent en dire les principaux acteurs du détachement d'intérimaires vers la Lorraine (représentants locaux des agences d'intérim luxembourgeoises, représentants syndicaux, responsables de l'inspection du travail française) (III).

\section{Le détachement en Saar-Lor-Lux}

Le détachement vers la France de travailleurs venus d'autres Étatsmembres de l'UE est en forte croissance. La Lorraine, qui appartient à la Grande Région européenne Saar-Lor-Lux, est l'un des territoires les plus concernés, sinon le plus concerné.

\section{I.1. La croissance des détachements intra-européens vers la France du Nord et de l'Est}

En 2000, les sections de l'inspection du travail française ont reçu 1443 déclarations de prestations de service international (PSI) ${ }^{3}$. Elles en reçoivent aujourd'hui 81420 (DGT, 2016:3).

Une même déclaration peut concerner plusieurs travailleurs. Ainsi, selon la DGT, le nombre de travailleurs détachés est passé de 7500 en 2000 à 286025 en $2015^{4}$, ce qui correspondrait à plus de 10,7 millions de jours détachés et à environ 46500 équivalents temps plein (ETP ; DGT, 2016). La durée moyenne des détachements en France est de 58 jours (DGT, 2016:13). Il y a donc aujourd'hui 38 fois plus de travailleurs détachés en France qu'en 2000. Encore doit-on ajouter qu'il s'agit là des travailleurs détachés déclarés. On le verra, les détachements non déclarés sont fréquents.

Selon cette même administration, la forte augmentation constatée s'expliquerait à la fois par une meilleure qualité de la mesure statistique, un meilleur respect de la réglementation relative au dépôt des déclarations et

3. Source : Direction générale du travail.

4. Tendance en hausse encore en 2016 avec 354151 salariés, soit $+24 \%$ par rapport à 2015 selon les données publiées par Le Monde le 11 novembre 2017. 
aussi à la généralisation des pratiques de détachement à l'ensemble des territoires qui donc, de ce fait, se normalisent. D'autres motifs peuvent être envisagés qui tiennent non seulement au contexte socioéconomique en Europe avec notamment le processus d'élargissement de l'UE aux pays de l'ancien bloc soviétique, mais également aux pratiques des acteurs du détachement.

Trois domaines d'activité économique sont particulièrement concernés (DGT, 2016:16) :

- le BTP, $27 \%$ des déclarations en 2015 ;

- l'intérim, $25 \%$ des déclarations ;

- et l'industrie, $16 \%$ des déclarations.

En 2015, un peu plus de 8 travailleurs détachés sur 10 sont ouvriers alors qu'ils étaient 3 sur 4 à appartenir à cette catégorie socioprofessionnelle en 2006 selon la DGT ${ }^{5}$.

Sept pays d'origine (Espagne, Pologne, Portugal, Allemagne, Roumanie et Luxembourg) totalisent $75 \%$ des déclarations reçues par les services de l'inspection du travail en 2015. Le Luxembourg pèse environ $8 \%$ de la totalité des déclarations faites en 2015. On le sait, ce petit État est situé au cœur de la Grande Région Sarre-Lorraine-Luxembourg, et frontalier à la fois de la Belgique, de l'Allemagne et de la France.

Le recours à ces travailleurs est fortement polarisé sur les régions frontalières de l'Hexagone. Pas loin de la moitié des déclarations de travail détaché en France en 2015 concerne des départements frontaliers. L'ancienne Région Lorraine enregistre 8935 déclarations, soit $11 \%$ de la totalité des déclarations. Elle figure au troisième rang des régions françaises. Elle a accueilli en 2015 un peu plus de 21100 travailleurs détachés (DGT, 2016:24) dont une part l'a été par des agences d'intérim localisées dans les pays frontaliers.

Les flux de travailleurs vers l'Ouest et le Nord de l'Europe, venant de l'Est et du Sud, seraient-ils devenus une dynamique centrale du fonctionnement du marché du travail ? L'effet de ces pratiques serait de baisser les niveaux de salaire dans les secteurs où ils sont nombreux. En attendant un « rééquilibrage » par le marché, les processus d'élargissement de l'UE (en particulier l'entrée au sein de l'Union européenne de pays de l'Est européen aux standards de travail et d'emploi et aux traditions syndicales bien différentes de celles de l'Europe de l'Ouest) auraient renforcé les clivages économiques et sociaux au sein de l'espace communautaire. L'adoption

5. On doit noter toutefois que la catégorie professionnelle est une variable très mal renseignée par les questionnaires que traitent les services de l'inspection du travail. 
en 2006 de la fameuse directive «Bolkestein ${ }^{6}$ qui organisait une libre prestation de services au sein du marché intérieur de l'Union européenne aurait bien sûr largement favorisé cette dynamique.

Dans un contexte d'européanisation et de mondialisation des économies, le recours au travail détaché serait le complément des délocalisations d'activité. On peut remarquer que les détachements de travailleurs ont principalement lieu dans les secteurs qui sont dans la nécessité de produire localement : bâtiment, tourisme, agriculture (Thoemmes, 2014). Pour les activités qui ne peuvent pas se délocaliser dans des pays à bas coût, dans les activités qui utilisent beaucoup de main-d'œuvre, le recours aux travailleurs détachés serait un autre moyen pour agir à la baisse sur les coûts de la main-d'œuvre.

\section{I.2. Saar-Lor-Lux : un territoire propice aux mobilités transfrontalières}

La Grande Région Saar-Lor-Lux est un territoire propice aux mobilités transfrontalières, en particulier sous détachement. De part et d'autre des frontières nationales, les dynamiques économiques et sociales y sont en effet très contrastées (tableau 1). Les différences entre les législations sociales et fiscales des États-membres de cette Grande Région sont profondes, en particulier entre le Luxembourg et ses voisins. Cela facilite le développement de mobilités transfrontalières et l'émergence de nouveaux intermédiaires de l'emploi.

Le Luxembourg est la principale destination des travailleurs frontaliers, qu'ils soient résidents en Allemagne, en Wallonie ou en Lorraine. Les travailleurs frontaliers y occupent $44 \%$ de l'emploi salarié intérieur. La Lorraine fait figure à l'inverse de principal « réservoir» de force de travail frontalière pour le Luxembourg et pour les Länder allemands. Plus de 100000 Lorrains (soit 1 actif sur 10) ont leur emploi de l'autre côté des frontières, dont les trois quarts au Luxembourg (et près de $20 \%$ en Allemagne).

$\mathrm{Au}$ nord et à l'est de la Lorraine, le phénomène est très marqué. Dans la zone d'emploi de Longwy, ancien bastion sidérurgique du nord de la Meurthe-et-Moselle et directement frontalier avec le Luxembourg et la Belgique, la moitié des actifs locaux a son emploi de l'autre côté de la frontière. Certaines petites communes directement frontalières ont entre 70 et $80 \%$ de leur population active occupée salariée par un employeur de l'autre côté des frontières (Belkacem, Pigeron-Piroth, 2012).

Les différentiels de coûts du travail, de niveaux de rémunérations, ou encore de coûts de l'immobilier, pour ne citer que ces seuls facteurs, alimentent le développement du travail frontalier sous toutes ses formes

6. Directive 2006/123/CE du Parlement européen et du Conseil du 12 décembre 2006 relative aux services dans le marché intérieur : Journal officiel n L 376 du 27 décembre 2006, p. 0036-0068, http://eur-lex.europa.eu/legal-content/FR/TXT/?uri=celex\%3A32006L0123. 
Tableau 1. Principales caractéristiques des différents territoires de la Grande Région en 2013

\begin{tabular}{|c|c|c|c|c|c|}
\hline & 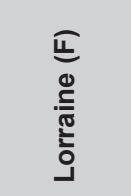 & 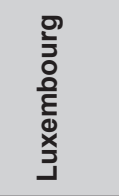 & 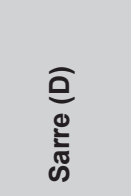 & 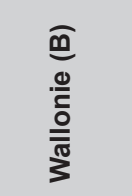 & 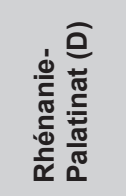 \\
\hline Superficie $\left(\mathrm{km}^{2}\right)$ & 23547 & 2586 & 2570 & 16844 & 19854 \\
\hline Population & 2350657 & 549680 & 990718 & 3576325 & 3994366 \\
\hline $\begin{array}{l}\text { Emploi intérieur } \\
\text { (lieu de travail) }\end{array}$ & 832399 & 386600 & 510000 * & 1227163 & 1923836 \\
\hline Agriculture (\%) & 2,1 & 1,2 & 0,5 & 1,5 & 2,2 \\
\hline Industrie (\%) & 15,6 & 9,5 & 22,5 & 12,5 & 19,7 \\
\hline Construction (\%) & 6,5 & 10,4 & 5,5 & 7,1 & 6,4 \\
\hline Services (\%) & 75,8 & 78,9 & 71,5 & 78,9 & 71,7 \\
\hline Ensemble (\%) & 100 & 100 & 100 & 100 & 100 \\
\hline $\begin{array}{l}\text { Population active totale } \\
\text { (au lieu de résidence) }\end{array}$ & 956217 & 235353 & 465200 & 1325377 & 1965900 \\
\hline Taux de chômage Eurostat (\%) & 12,2 & 5,8 & 6,2 & 16,1 & 4,1 \\
\hline $\begin{array}{l}\text { Revenu disponible brut } \\
\text { des ménages } \\
\text { (en euros par habitant) }\end{array}$ & 18663 & 32107 & 19421 & 17009 & 21608 \\
\hline
\end{tabular}

*En 2012.

Source : Offices statistiques de la Grande Région (2014).

(Belkacem, Pigeron-Piroth, 2011). Les salaires sont plus élevés au Luxembourg, les prestations sociales plus avantageuses, la fiscalité plus attractive, les minima salariaux plus incitatifs. Un travailleur intérimaire détaché du Luxembourg en Lorraine bénéficie du régime luxembourgeois.

Mais ces frontaliers recrutés par une agence de travail temporaire basée au Luxembourg peuvent être envoyés en mission en Lorraine. Français résidents en Lorraine, ils restent considérés comme des travailleurs frontaliers puisque leur employeur est situé au Luxembourg. Ils résident et travaillent en France sans avoir à passer la frontière au sens du règlement européen (CE) n 883/2004. Mais tout leur régime fiscal et de protection sociale reste de droit luxembourgeois.

\section{I.3. L'intérim transfrontalier : une pratique ancienne capable de se réorienter en fonction des changements règlementaires}

Au sein de l'espace Saar-Lor-Lux, l'intérim transfrontalier se pratique depuis longtemps. Dans les années 1970 déjà, les agences d'intérim 
envoyaient en mission en Sarre des travailleurs lorrains. Les intérimaires transfrontaliers représentaient environ $10 \%$ des flux de frontaliers entre ces deux régions. C'était une main-d'œuvre plutôt qualifiée, travaillant dans les métiers de la construction. On les nommait à l'époque « travailleurs de location »(Belkacem, Pigeron-Piroth, 2012). L'élément nouveau est aujourd'hui l'essor important de l'intérim transfrontalier entre le Luxembourg et la Lorraine, sur des missions proposées par les agences luxembourgeoises.

Les agences d'intérim se sont positionnées au sein de cet espace transfrontalier comme des acteurs incontournables en matière de gestion des ressources humaines. Elles ont étoffé leurs offres de services et proposent aujourd'hui des prestations très diversifiées comme l'aide à l'insertion professionnelle, la recherche de qualifications pointues, le conseil, le portage salarial et même la formation. Elles ont su nouer des relations durables avec beaucoup de leurs clients, même ceux situés de l'autre côté des frontières. Ces clients sont souvent des établissements de grands groupes ou de leurs sous-traitants. Les entreprises de travail temporaire cherchent à nouer des relations durables avec eux. Ces relations se développent en fonction des besoins spécifiques de chacun des clients. Ce sont en quelque sorte des véritables stratégies commerciales individualisées.

Les Lorrains frontaliers sont souvent inscrits dans les agences luxembourgeoises et envoyés en mission au-delà des frontières luxembourgeoises, en Lorraine par exemple. En 2005, 18 \% d'entre eux étaient détachés de l'autre côté de la frontière ; 25 \% quatre ans plus tard (Statec, 2012). On notera toutefois que le règlement européen (CE) n 883/2004, qui coordonne les systèmes de Sécurité sociale et règlemente entre autres le temps de travail passé à l'étranger, a contribué à réduire sensiblement la proportion de travailleurs détachés du Luxembourg vers les territoires limitrophes (Statec, 2012). Entre 2009 et 2011, la part des intérimaires inscrits au Luxembourg et détachés à l'étranger serait ainsi passée de $25 \%$ à moins de $9 \%$ (Statec).

Les dernières données publiées par la Direction générale des entreprises, de la concurrence, de la consommation, du travail et de l'emploi (Direccte, 2015) concernant les prestations de service internationales (PSI) montrent cependant que ce phénomène est loin d'être marginal, si on le place à l'échelle de l'ensemble des détachements effectués en Lorraine. Pour les quatre départements lorrains, ce sont 7371 demandes de PSI qui ont été faites en 2014. Les deux tiers (65\%, soit 4761 déclarations de PSI) émanent des seules entreprises localisées au Luxembourg. Plus du tiers (2668 déclarations, 36,2\%, représentant un peu plus de 5000 intérimaires) sont le fait des seules entreprises de travail temporaire luxembourgeoises. 


\section{Les fondements économiques et sociaux du détachement de travailleurs intérimaires du Luxembourg vers la Lorraine}

Les entreprises utilisatrices lorraines recourent aux services des agences d'intérim luxembourgeoises souvent situées à seulement quelques kilomètres de l'autre côté de la frontière. Le Luxembourg dispose en effet d'un net avantage sur ses voisins européens pour des raisons institutionnelles. Le coût patronal du travail y est comparativement plus faible et les niveaux de salaires y sont à l'inverse relativement plus élevés. Le Luxembourg apparaît ainsi à la fois attractif pour les entreprises (particulièrement dans les secteurs de l'industrie et de la construction, gros utilisateurs d'intérimaires) et pour les travailleurs.

En ce domaine, la petite taille du Luxembourg au cœur de la Grande Région Saar-Lor-Lux n'est pas indifférente. L'adaptation du pays aux évolutions économiques et sociales en serait facilitée. Dans un si petit État, les choix politiques pourraient être pris plus rapidement mais aussi plus facilement. D'autant que ces choix paraissent soutenir les avantages du Luxembourg par rapport à ses différents voisins européens, dont la France, concernant le régime de financement de la protection sociale, le système fiscal, les politiques de rémunération. Ils entretiennent l'attractivité de ce petit État relativement à ses grands voisins pour attirer à la fois les travailleurs et les entreprises.

Plusieurs éléments contribuent ainsi aux avantages de coût de la maind'œuvre du Luxembourg.

\section{II.1. Le système social luxembourgeois est moins financé par les cotisations sociales}

Au Luxembourg, les régimes obligatoires de sécurité sociale ont deux principales sources de financement : les cotisations sociales des salariés et des employeurs et les contributions des pouvoirs publics (grâce aux impôts). Selon une étude d'Eurostat (2017), les cotisations sociales (employeurs et salariés) participent à hauteur d'un peu plus de la moitié au Grand-duché de Luxembourg au financement de la protection sociale en 2014. Alors qu'en France, elles y participent à presque les deux tiers, soit nettement davantage. C'est ce que confirme un rapport de la Direction de la recherche, des études, de l'évaluation et des statistiques (Drees, 2017:51), qui indique que les contributions publiques de l'État participent à 11,2\% des ressources qui servent à financer la protection sociale. Ce rapport relève en même temps une baisse tendancielle du poids des cotisations sociales dans le financement de la protection sociale avec le développement d'impôts et de taxes affectés (CSG, CRDS). À la différence des entreprises lorraines, les entreprises luxembourgeoises contribuent moins 
Tableau 2. Taux de cotisations sociales au Luxembourg en 2017

En \% (sauf mention contraire)

\begin{tabular}{|l|c|r|r|}
\hline & Salarié & \multicolumn{1}{|c|}{ Employeur } & Plafond (en $€$ ) \\
\hline Maladie - Maternité & & & \\
\hline Prestations en nature & 2,80 & 2,80 & 9992,93 \\
\hline Prestations en espèces & 0,25 & 0,25 & 9992,93 \\
\hline Mutualité des employeurs (MDE) & $/$ & Entre 0,51 et 2,92 & 9992,93 \\
\hline Vieillesse Invalidité Survivants & 8,00 & 8,00 & 9992,93 \\
\hline Dépendance & 1,40 & $/$ & Totalité du salaire \\
\hline Assurance accident & $/$ & 1,00 & 9992,93 \\
\hline Santé au travail & $/$ & 0,11 & 9992,93 \\
\hline
\end{tabular}

Sources : CLEISS, Centre commun de la Sécurité sociale (CCSS) : http://www.cleiss.fr/docs/cotisations/ luxembourg.html.

Tableau 3. Taux de cotisations sociales en France en 2017

En \% (sauf mention contraire)

\begin{tabular}{|c|c|c|c|}
\hline & Salarié & Employeur & Plafond (en $€$ ) \\
\hline Ass. Maladie, Maternité, Invalidité, Décès & 0,75 & 12,89 & Sur le total du salaire \\
\hline Contribution solidarité autonomie (CSA) & 1 & 0,30 & Sur le total du salaire \\
\hline Assurance vieillesse plafonnée & 6,90 & 8,55 & 3269 \\
\hline Assurance vieillesse & 0,40 & 1,9 & Sur le total du salaire \\
\hline Accidents du travail & 1 & Variable & Sur le total du salaire \\
\hline Cotisation pénibilité de base & I & 0,01 & Sur le total du salaire \\
\hline Allocation famille & 1 & 5,25 ou 3,45 & Sur le total du salaire \\
\hline Contribution sociale généralisée (CSG) & 7,50 & l & $98,25 \%$ du sal. brut \\
\hline $\begin{array}{l}\text { Contribution à la réduction } \\
\text { de la dette sociale (CRDS) }\end{array}$ & 0,50 & l & $98,25 \%$ du sal. brut \\
\hline Chômage & 2,40 & 4,05 & 13076 \\
\hline Régime de garantie des salaires (AGS) & l & 0,15 & 13076 \\
\hline \multicolumn{4}{|l|}{ Retraite complémentaire } \\
\hline \multicolumn{4}{|l|}{$\begin{array}{l}\text { Non cadres (Association des régimes } \\
\text { de retraite complémentaire, ARRCO) }\end{array}$} \\
\hline Tranche 1 & 3,10 & 4,65 & 3269 \\
\hline $\begin{array}{l}\text { Association pour la gestion du fonds } \\
\text { de financement (AGFF) tranche } 1\end{array}$ & 0,80 & 1,20 & 3269 \\
\hline Tranche 2 & 8,10 & 12,15 & De 3269 à 9807 \\
\hline AGFF tranche 2 & 0,90 & 1,30 & De 3269 à 9807 \\
\hline
\end{tabular}

Sources : CLEISS, Centre commun de la Sécurité sociale (CCSS) : http://www.cleiss.fr/docs/regimes/ regime_francea2.html. 
à la protection sociale, qui est davantage partagée entre tous les assujettis à l'impôt, d'où un coût du travail plus faible. Autre différence de taille : les cotisations sociales apparaissent moins nombreuses au Luxembourg comme le montrent les tableaux 2 et 3 .

Une étude du Service central de la statistique et études économiques au Luxembourg (Statec, 2014) montre clairement l'avantage compétitif de ce pays par rapport à ses voisins frontaliers en matière de coût de la maind'œuvre. Le coût horaire moyen de la main-d'œuvre s'élève à 33,91 euros au Luxembourg contre 34,25 euros pour la France en 2012. Ces écarts sont, en revanche, plus importants dans les secteurs d'activité économique de l'industrie et de la construction, là où sont utilisés en plus grand nombre les travailleurs intérimaires. Pour les secteurs de l'industrie et de la construction considérés ensemble, le coût horaire moyen s'établit à seulement 30,7 euros au Luxembourg, soit presque 6 euros de moins par rapport à la France (36,4 euros).

\section{Graphique 1. Coût horaire moyen de la main-d'œuvre * par secteur en Grande Région en 2012}

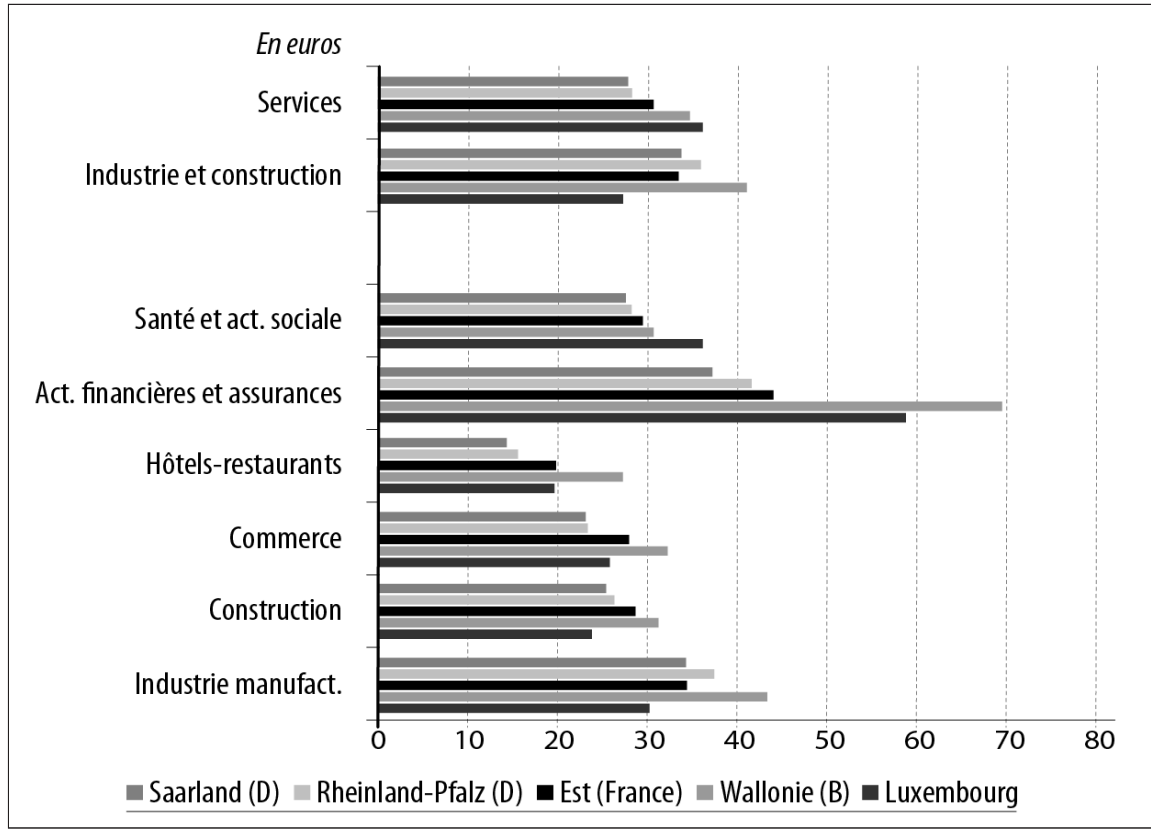

* Le coût annuel de la main-d'œuvre couvre tous les coûts liés au travail : rémunérations brutes régulières mais aussi irrégulières (bonus de fin d'année, $13^{\mathrm{e}}$ mois, indemnités de licenciement, etc.), avantages en nature, charges patronales, autres coûts indirects (coûts de formation, frais de recrutement, etc.) de l'ensemble des salariés (qu'ils soient couverts par une convention collective ou non). Le coût horaire est égal au coût annuel divisé par le nombre d'heures effectivement travaillées (Statec, 2014).

Source : Statec, Eurostat (enquête Coût de la main-d'œuvre 2012), en équivalents temps plein, inclus les apprentis. 
En effet, le coût de la main-d'œuvre se répartit en différentes composantes : celle des salaires et traitements bruts, celle des cotisations sociales à la charge de l'employeur, et celle relative à d'autres dépenses directement liées à la main-d'œuvre supportées par les employeurs. Cette dernière composante comprend les frais de formation professionnelle, d'autres dépenses de l'employeur ou encore des taxes et impôts à la charge de ce dernier, déductions faites des subventions reçues par celui-ci. La part des cotisations salariales à la charge des employeurs s'élève au Luxembourg à environ $13 \%$ du coût total de la main-d'œuvre, soit presque trois fois moins qu'en France (33,1 \%) (Insee, 2015). Le Luxembourg dispose ainsi d'un important avantage compétitif sur la France, du moins dans les secteurs économiques qui font le plus appel à l'intérim.

\section{II.2. Les prestations sociales et les salaires minimaux sont plus élevés au Luxembourg}

Un autre avantage expliquant l'attractivité qu'exerce le Luxembourg sur ses voisins et plus particulièrement pour les travailleurs concerne l'important différentiel des prestations sociales et des salaires minimaux (tableau 4). Même s'ils résident en Lorraine, les travailleurs détachés bénéficient de ces prestations sociales, par exemple les allocations familiales, en fonction du nombre d'enfants dans le ménage. En début de carrière professionnelle, ils bénéficient également des salaires minimaux selon qu'ils sont qualifiés ou non.

Les niveaux de salaires pratiqués au Luxembourg sont relativement élevés comparés à la France. Selon les données, certes très parcellaires, existantes sur le sujet, un couvreur en début de carrière gagne au Luxembourg 19822 euros par an. Il peut atteindre avec 10 ans d'expérience jusqu'à 27000 euros (Association des travailleurs frontaliers - frontaliers.lu, 2017). Selon Bati-Actu Emploi, le salaire pratiqué en région Grand-Est en France pour ce même métier s'établit à seulement 16849 euros par an. Nous observons ces mêmes différences pour d'autres métiers du BTP sur lesquels les travailleurs sont fréquemment détachés comme celui de peintre en bâtiment (19826 euros et 16852 euros respectivement au Luxembourg et dans la Région Grand-Est en France) ou encore comme celui de conducteur d'engins (25600 et 21760). Ces différences peuvent également s'expliquer par des stratégies de captation de la main-d'œuvre qualifiée transfrontalière sur des métiers en tension. Des salaires élevés contribuent en effet à stabiliser et à fidéliser la main-d'œuvre qualifiée.

\section{II.3. Le système fiscal luxembourgeois de prélèvement à la source}

Le Luxembourg dispose également d'un autre avantage de poids. Il s'agit du niveau des impôts là encore plus incitatif pour les travailleurs. 
Tableau 4. Allocations familiales et salaire minimum au Luxembourg * et en France ${ }^{* *}$ en 2017 : des montants très différents

En euros

\begin{tabular}{|l|r|r|}
\hline & Luxembourg & France \\
\hline Allocations familiales & 265,00 & 129,86 \\
\hline Pour 1 enfant & 594,48 & 296,23 \\
\hline Pour 2 enfants & 1033,38 & 462,61 \\
\hline pour 3 enfants & 1472,08 & 9,76 brut horaire \\
\hline Pour 4 enfants & 13,86 & 1480,27 brut mensuel \\
\hline Salaire minimum qualifié & 2398,30 & \\
\hline Brut horaire & & \\
\hline Brut mensuel & 11,55 & \\
\hline Salaire minimum non qualifié & 1998,59 & \\
\hline Brut horaire & & \\
\hline Brut mensuel & & \\
\hline
\end{tabular}

* Pour tous les enfants nés après le $1^{\text {er }}$ août 2016 au Luxembourg, pour les parents qui ont commencé à travailler au Luxembourg après le $1^{\text {er }}$ août 2016 , et pour les personnes avec enfants qui se sont installées au Luxembourg après le $1^{\text {er }}$ août 2016, une allocation unique de 265 euros par enfant est attribuée.

** En France, les allocations familiales sont modulées en fonction des ressources du ménage. Ces données sont établies sur la base de ressources ne dépassant pas 67408 euros par an, une situation qui correspond à la majorité des travailleurs détachés via l'intérim. Au-delà de ce niveau, ces prestations diminuent.

Sources : Guichet public.lu (2017), CAF.fr (2017).

Les travailleurs détachés en Lorraine paient leur impôt sur le revenu au Luxembourg. Celui-ci est directement prélevé à la source, c'est-à-dire chaque mois sur leur salaire. Il est donc moins visible. C'est surtout là que réside l'avantage du système fiscal luxembourgeois mis en avant par les travailleurs enquêtés.

Par ailleurs, le système de l'impôt sur le revenu avantage surtout les personnes qui bénéficient de revenus élevés au Luxembourg comparativement à celles qui paient leurs impôts en France. Le Luxembourg a opté pour un modèle de calcul proche de celui de la France mais avec une plus forte progressivité de l'impôt, comme le montre le tableau 5.

Par ailleurs, en France, un système de décote est appliqué à l'impôt en fonction de la situation de la personne : par exemple 1552 euros en 2016 si la personne vit seule et 2559 euros pour un couple avec ou sans enfants. Un nombre de parts est retenu selon le nombre d'enfants dans le ménage qui rend l'impôt plus proportionnel en fonction de la configuration du ménage. Le Luxembourg distingue, lui, trois classes d'imposés : 
- la classe 1 concerne les célibataires, divorcés ou séparés. Le barème s'applique sur la totalité du revenu imposable ;

- la classe 1a se rapporte aux personnes non mariées avec enfants ou les veufs. Pour tous les revenus inférieurs à 45000 euros, l'impôt se calcule sur la totalité du revenu ;

- la classe 2 rassemble les personnes mariées ou liées par un contrat de partenariat (avec ou sans enfants). Le barème est appliqué sur la moitié du revenu pour chaque membre du ménage.

Tableau 5. Les barèmes de l'impôt sur le revenu au Luxembourg et en France en 2017

\begin{tabular}{|c|c|c|c|}
\hline \multicolumn{2}{|c|}{ Au Luxembourg } & \multicolumn{2}{|c|}{ En France } \\
\hline $\begin{array}{l}\text { Revenus } \\
\text { (en } €)\end{array}$ & $\begin{array}{l}\text { Taux d'imposition } \\
\text { (en \%) }\end{array}$ & $\begin{array}{l}\text { Revenus } \\
\text { (en } €)\end{array}$ & $\begin{array}{c}\text { Taux d'imposition } \\
\text { (en \%) }\end{array}$ \\
\hline Inf. à 11265 & 0 & inf. à 9710 & 0 \\
\hline De 11265 à 13173 & 8,56 & 9700 à 26818 & 14 \\
\hline De 13173 à 15081 & 10,70 & 26818 à 71898 & 30 \\
\hline De 15081 à 16989 & 12,84 & 71826 à 152260 & 41 \\
\hline De 16989 à 18897 & 14,98 & Sup. à 152260 & 45 \\
\hline De 18897 à 20805 & 17,12 & & \\
\hline De 20805 à 22713 & 19,26 & & \\
\hline De 22713 à 24621 & 21,40 & & \\
\hline De 24621 à 26529 & 23,54 & & \\
\hline De 26529 à 28437 & 25,68 & & \\
\hline De 28437 à 30345 & 27,82 & & \\
\hline De 30345 à 32253 & 29,96 & & \\
\hline De 32253 à 34161 & 32,10 & & \\
\hline De 34161 à 36069 & 34,24 & & \\
\hline De 36069 à 37977 & 36,38 & & \\
\hline De 37977 à 39885 & 38,52 & & \\
\hline De 39885 à 41793 & 40,66 & & \\
\hline De 41793 à 100000 & 41,73 & & \\
\hline De 100000 à 150000 & 42,80 & & \\
\hline Sup. à 150000 & 43,63 & & \\
\hline
\end{tabular}

Source: impôts direct.public.lu - https://www.service-public.fr/particuliers/vosdroits/F1419. 
Par exemple, pour une personne qualifiée, mariée, couvreur de métier, deux enfants, ayant un revenu annuel de 30000 euros (située donc en classe 2), l'impôt sur le revenu s'élève à 634 euros (soit environ 53 euros prélevés par mois) ${ }^{7}$. Si la personne est en classe 1 (célibataire sans enfant), l'impôt s'élève à 2791 euros (soit 232,6 euros par mois). En France, pour une même situation civile, familiale et un même niveau de revenu imposable, le travailleur ne paiera pas d'impôts. S'il est célibataire, sans enfant, son impôt s'élèvera par contre à 630 euros annuels en France.

\section{II.4. Le système de retraite luxembourgeois est également plus avantageux}

À partir du moment où ils ont cotisé pendant au moins un an, les travailleurs détachés du Luxembourg vers la Lorraine bénéficient d'une retraite du Luxembourg. Les caisses de retraite française et luxembourgeoise se coordonnent pour calculer le montant de la pension selon les périodes de cotisation dans chacun des pays (voire dans d'autres pays frontaliers comme en Belgique ou en Allemagne) comme il est fréquent en région frontalière. Pour le Luxembourg, il s'agit souvent d'une retraite additionnelle que le travailleur ne perçoit qu'après avoir atteint l'âge légal de départ à la retraite, soit 65 ans, et même s'il est déjà en retraite en France où l'âge légal commence à 60-62 ans. Au Luxembourg, la pension de retraite se compose d'un montant fixe qui est un forfait de $1 / 40^{\mathrm{c}}$ acquis par année de cotisation dans la limite maximale de 40 années) et d'un montant variable correspondant à un pourcentage des salaires perçus durant la trajectoire professionnelle au Luxembourg. Et ces salaires sont généralement supérieurs de l'ordre de $20 \%$ à $50 \%$ selon les responsables d'agences d'intérim que nous avons rencontrés. Pour un salarié qui a cotisé pendant 40 années au Luxembourg, sa pension ne peut être inférieure à $90 \%$ du montant de référence soit 1771,75 euros (au $1^{\text {er }}$ janvier 2017). Si le travailleur n'a cotisé que quelques années au Luxembourg, la pension additionnelle est réduite de $1 / 40^{\mathrm{e}}$ pour chaque année manquante. Si la période de cotisation au Luxembourg est inférieure à un an, celle-ci, même si elle n'ouvre pas droit à pension au Luxembourg, est néanmoins prise en compte par la caisse compétente pour la retraite en France.

En définitive, le lieu géographique de signature du contrat de travail, au Luxembourg ou en Lorraine, est décisif aussi bien pour les travailleurs qui percevront des revenus plus élevés que pour les employeurs qui bénéficieront de coûts de la main-d'œuvre moins élevés. L'égalité de traitement sur le lieu de travail ne joue pas ici.

7. Ces calculs ont été réalisés à partir de logiciels en ligne des services publics luxembourgeois (http://www.impotsdirects.public.lu/fr/baremes/personnes-physiques.html) et français (https:// www3.impots.gouv.fr/simulateur/cgi-bin/calc-2017.cgi). 
Pour comprendre cela, prenons un cas précis. Un couvreur appartenant à un ménage avec deux enfants qui gagne 2500 euros brut ne disposera pas du même salaire net selon que son contrat de travail est de droit luxembourgeois ou français. Au Luxembourg, il percevra un salaire net après impôt (comme celui-ci est prélevé à la source) de 2232,45 euros contre seulement 1923,15 euros en France. Le travailleur en France devra encore payer l'impôt sur le revenu. Si nous tenons compte du nombre légal d'heures hebdomadaires de travail inférieur en France ( 35 heures) par rapport au Luxembourg (40 heures), la différence s'atténue quelque peu mais reste significative. De plus, le couvreur bénéficiera en plus de son salaire de prestations sociales pour ses deux enfants (les allocations familiales) plus généreuses au Luxembourg (595 euros) qu'en France (130 euros) ${ }^{8}$.

\section{Qui sont les intérimaires transfrontaliers ?}

Les informations disponibles sur le détachement transfrontalier d'intérimaires en Lorraine (principalement les déclarations de prestations de service international, les PSI) sont trop insuffisantes pour aider à préciser la dynamique du phénomène et à comprendre le débat. En particulier, le profil socioéconomique de ces intérimaires détachés reste mal connu. L'inspection du travail française fait état dans ses documents internes de quelques informations non publiées. Elles montrent, on l'a dit, que les

\section{Graphique 2. Catégorie socioprofessionnelle des salariés détachés en Lorraine en 2014}

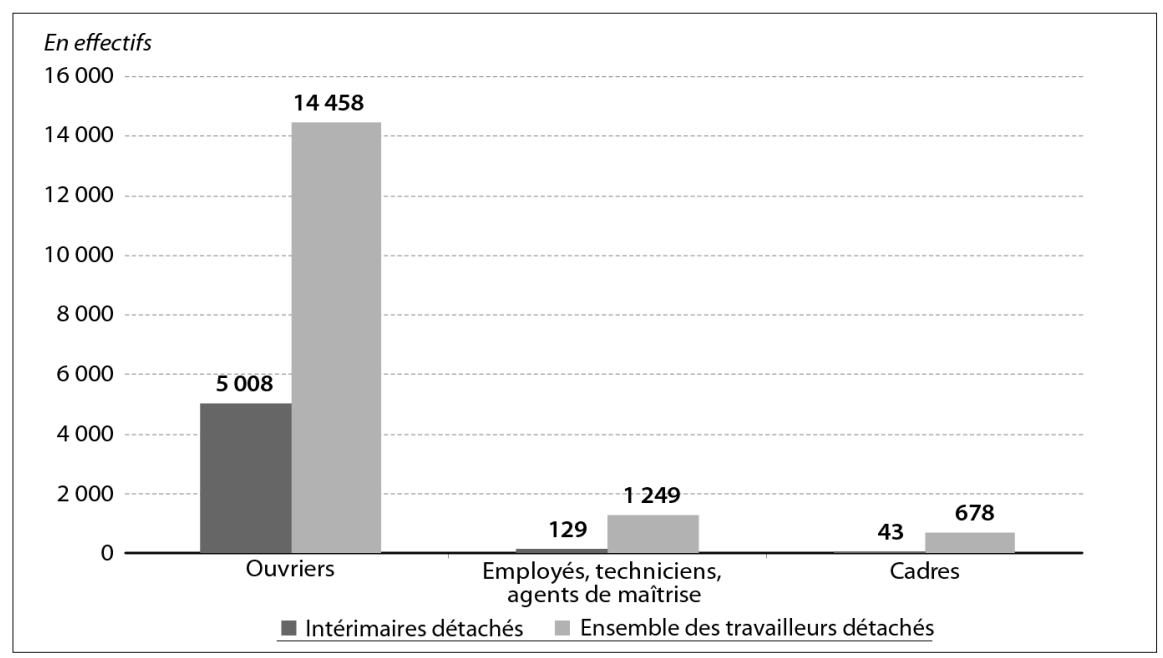

Source : Direccte Lorraine, document interne (2015).

8. Ces calculs ont été réalisés à partir d'utilitaires (logiciels accessibles en ligne - association travailleursfrontaliers.lu et Cogiloc.com pour la France). 
travailleurs détachés en Lorraine, et plus encore les détachés sur une mission d'intérim, sont quasi exclusivement des personnels hommes $(88 \%)$ et ouvriers ( $97 \%$; graphique 2$)$.

Nous avons donc tenté de compléter les données et documents disponibles par quelques entretiens d'intérimaires, sept personnes qui travaillent en mission d'intérim en France (encadré 2).

Ces entretiens donnent quelques enseignements utiles. Les intérimaires détachés en France que nous avons interviewés sont des travailleurs plutôt qualifiés, appartenant au noyau dur des intérimaires fidélisés par leur agence, donc plutôt le segment des intérimaires professionnels, davantage qualifiés, bénéficiant de missions plus longues que les autres intérimaires et de meilleures conditions de travail (Kornig, 2007). Un seul des sept intérimaires enquêtés n'a pas de qualification spécifique (Manuel). La formation qu'il a reçue est peu utile pour les emplois qu'il peut trouver. Mais il revendique beaucoup d'expérience : «Mon Bac pro administratif ne me sert pas dans l'intérim. Je n'arrive pas à trouver des missions dans ce domaine. Et dans les métiers des chantiers, je n'ai pas de qualification mais j'ai beaucoup d'expérience comme manœuvre. »

\section{Encadré 2}

\section{Sept entretiens d'intérimaires}

L'accès au terrain s'est révélé un exercice complexe. Nous avons ainsi été confrontés à la réticence manifeste, voire parfois au refus, des acteurs d'échanger autour des pratiques de détachement. Bien que légale, cette forme de mobilité et de mobilisation de la main-d'œuvre reste entourée d'un halo qui voit se mêler crainte des travailleurs, prise de distance des acteurs collectifs censés les représenter et des entreprises de travail temporaire. Les résultats de cette enquête qui sont ici partiellement mobilisés ne prétendent pas à l'exhaustivité. Ils sont utilisés ici avant tout comme un éclairage inédit d'une modalité de détachement professionnel spécifique dans un environnement socioéconomique particulier, celui de territoires frontaliers.

1. Enriko est conducteur d'engin. II a un permis poids lourd (12 tonnes). Divorcé avec un enfant, il a 35 ans. II vit en Lorraine depuis une vingtaine d'années dans le département de la Moselle dans une ville frontalière du Luxembourg. Du fait de ses qualifications, il se voit régulièrement proposer des missions de longue durée de l'autre côté des frontières, principalement en Lorraine. Très polyvalent, Enriko détient des compétences dans différents domaines : électricité, carrelage et conduite d'engins. Ceci lui assure une relative continuité des missions même si elles ne correspondent pas toujours à son niveau de qualification. C'est à la suite d'un licenciement récent 
(en 2013) qu'Enriko s'est orienté vers l'intérim au Luxembourg. Pour lui, les avantages du détachement sont nombreux : les salaires sont plus élevés grâce aux paniers repas et indemnités de déplacement. Lorsque les missions sont éloignées de son domicile, il habite dans des hébergements collectifs. II considère être bien traité sans discrimination par rapport aux autres salariés ou par rapport aux intérimaires nationaux. Enfin, il considère ses conditions de travail comme étant tout à fait normales.

2. Michel est spécialisé dans le domaine de la mécanique. II a un CAP, un BEP et un Bac pro. Divorcé, également avec deux enfants, il a 47 ans. II vit aussi en Lorraine dans le département de la Moselle. Il est aussi régulièrement détaché sur des missions de longue durée, souvent en Lorraine. Avec sa femme, il tenait un commerce de fleurs. Des accidents de la vie (difficultés personnelles, divorce...) l'ont amené à l'intérim en 2000. Le mode de fonctionnement de l'intérim correspond selon lui à ses aspirations même si les missions ne correspondent pas toujours à son niveau de qualification. Pour lui, les avantages de l'intérim et du détachement s'expliquent par des raisons tout autres comme le système de mensualisation des impôts (prélèvement à la source) et les montants des allocations familiales. Comme Enriko, Michel considère être bien traité sans aucune discrimination par rapport aux autres salariés ou par rapport aux intérimaires nationaux dans l'entreprise utilisatrice.

3. Manuel est âgé de 23 ans. II est célibataire et vit dans une petite commune de Meurthe-et-Moselle frontalière du Luxembourg. Il est chauffeur-livreur en temps normal et vient à l'intérim de manière irrégulière ou occasionnelle. II a un niveau Bac dans le domaine administratif (Bac pro), une qualification qu'il n'arrive cependant pas à monnayer dans l'intérim. Aussi accepte-t-il des missions en tant que manutentionnaire ou chauffeur-livreur. Les missions qu'on lui propose sont de durée moyenne (environ trois mois) mais relativement continues. II a déjà réalisé des missions même en dehors de la Lorraine. II a été détaché sur des chantiers en Normandie (Cherbourg), nous expliquait-il. Hormis le fait que l'intérim lui permet de travailler, Manuel vit ce statut plus comme une contrainte que comme un choix délibéré. En tant que manutentionnaire, les missions proposées sont peu valorisantes et les salaires plutôt faibles même s'il considère être bien traité avec des conditions de travail correctes.

4. Comme Michel, Christian est qualifié dans le domaine de la mécanique. Marié, un enfant, il a 35 ans. II a un Bac sciences et technologies industrielles (STI) et a travaillé pendant cinq ans dans la marine marchande. II habite en Meurthe-et-Moselle dans une toute petite commune frontalière du Luxembourg. Il est régulièrement détaché en Lorraine. Mais les missions, même si elles correspondent le plus souvent à son niveau de qualification, sont de durées très irrégulières, pouvant aller de quelques semaines à plusieurs mois. C'est à la suite d'un conflit social (grève SNCM) que Christian est venu à l'intérim. C'est un choix délibéré. C'est une formule de travail qui correspond comme pour Michel à ses aspirations de liberté à l'égard du travail et surtout des marges de liberté qu'elle autorise par rapport 
à la subordination à un seul patron. Pour Christian, les avantages de l'intérim au Luxembourg et du détachement sont nombreux : des salaires plus élevés qu'en France et la mensualisation des impôts. Comme pour les travailleurs intérimaires précédents, Christian n'a pas de soucis particuliers tant en termes de traitement individuel que de conditions de travail.

5. Nordine habite en Meurthe-et-Moselle tout proche du Luxembourg. Âgé de 27 ans, pacsé, sans enfant, il est cariste de profession (niveau CASES 3). II est venu à l'intérim très récemment (en 2014). II a eu une trajectoire professionnelle très accidentée. II était d'abord hôte de caisse, puis vendeur dans différents magasins de sport et enfin cariste dans des grandes entreprises régionales. C'est le statut attractif de travailleur frontalier du point de vue des rémunérations et des impôts qui a poussé Nordine à s'inscrire dans une agence d'intérim au Luxembourg. Les missions proposées sont régulières mais pour des durées variables, généralement des missions de quelques semaines avec renouvellements successifs. S'il considère qu'il est globalement bien traité en tant qu'intérimaire par ses chefs, Nordine estime qu'il reste des points à améliorer sur le plan de la sécurité.

6. Âgé de 42 ans, marié, un enfant, Norbert est également régulièrement détaché en Lorraine mais sur des missions relativement longues (en moyenne 5-6 mois avec renouvellement). Comme Enriko, il a plusieurs compétences : soudeur, chalumiste, monteur. Cette multi-compétence lui permet d'élargir les possibilités de missions aussi bien au Luxembourg que de l'autre côté des frontières. Mais ces missions ne correspondent pas toujours à son vrai niveau de qualification. II a obtenu la licence TIG/Arc semi en soudure. II est venu à l'intérim en 2000 et ce statut d'emploi correspond à ses attentes. Pour Norbert, l'intérim au Luxembourg est une formule de travail avantageuse, du point de vue des salaires, des impôts avec le système de mensualisation et aussi des retraites. Norbert considère que les conditions de travail sont plutôt bonnes et qu'il est plutôt bien traité par ses supérieurs hiérarchiques, mais il reconnaît qu'il peut être mal considéré par les autres salariés de l'entreprise utilisatrice en raison de son statut de travailleur détaché.

7. Pierre a 50 ans. Marié, un enfant, il habite en Moselle pas loin du Luxembourg. II détient également plusieurs qualifications (monteur, chalumiste...), notamment en soudure où il a obtenu comme Norbert une licence soudure en Arc semi. Les missions qu'on lui propose sont plutôt longues (8 mois en moyenne). II est souvent détaché aussi bien en Lorraine qu'en Wallonie (Belgique). II a commencé dans l'intérim en 2006 et s'est spécialisé dans la soudure. Il est régulièrement appelé par les mêmes entreprises dont certaines ont même cherché à le recruter. L'intérim a été pourtant un choix forcé pour lui. C'est à la suite de son licenciement qu'il a décidé de s'inscrire dans une agence d'intérim à Luxembourg sur les conseils de ses amis. Comme pour Enriko, pour Pierre, les avantages de l'intérim résident non seulement dans des salaires comparativement plus élevés qu'en France mais aussi dans différents suppléments comme les indemnités de déplacement ou encore les paniers repas. Pierre déclare également être bien traité par ses chefs et trouve les conditions de travail tout à fait correctes. 
Pour les intérimaires qui disposent de plusieurs qualifications, les possibilités de missions sont non seulement plus nombreuses et régulières mais les durées de mission sont aussi plus longues. L'un d'eux juge que la multiqualification est un sérieux avantage pour l'intérim. Cela autorise en plus une certaine forme de liberté, liberté de choisir, de décider quand travailler : «Je n’ai pas de problème de missions. Je peux même les choisir. Je sais quand prendre mes vacances » (Norbert).

Pour tous les intérimaires enquêtés, la durée moyenne des missions au Luxembourg est nettement plus importante qu'en France (plusieurs mois contre un peu moins de deux semaines en moyenne). Mais avant d'être détachés, les intérimaires sont testés sur de petites missions. Ce que confirme l'un des responsables d'agence que nous avons rencontré : "Au départ c'est des petites missions qu'on propose pour connaitre l'intérimaire. Elles sont ensuite prolongées sur plusieurs mois si les gars sont fiables, sont compétents. »

Ceci étant, les missions ne correspondent pas toujours au niveau de qualification des intérimaires détachés. Elles peuvent être très variables, souvent de niveaux inférieurs. «Les missions qu'on me confie sont de qualifications très variables. Elles peuvent correspondre à mes qualifications, mais peuvent être aussi inférieures et même parfois supérieures. Elles sont souvent inférieures. Il m'est arrivé une fois d'avoir une belle mission dans une entreprise qui faisait de la petite mécanique, ce qui correspondait parfaitement à mon diplôme d'électromécanicien » (Michel).

Le lieu de la mission est également très variable. Ce sont souvent les grandes entreprises appartenant à de grands groupes implantés en Lorraine qui font appel à des intérimaires inscrits au Luxembourg. Mais la mission peut également concerner des entreprises localisées beaucoup plus loin, en Normandie par exemple. L'un des intérimaires s'est vu confier des missions de manœuvre sur un gros chantier situé à Cherbourg. Il l'a très mal vécu. "L'intérim ça a des avantages, c'est vrai, pour travailler, mais aussi beaucoup d'inconvénients comme devoir aller travailler très loin. $\mathrm{Si}$ on refuse, on n'a plus de mission... Personnellement, je préfère des missions sans grand déplacement» (Manuel). D’autres semblent avoir davantage intériorisé les injonctions de mobilité. «Si j’accepte un travail loin de chez moi, c'est pour montrer aussi ma capacité à être mobile, que je suis prêt à me déplacer loin. Ça plaît... et il y a les défraiements qui tombent en plus du salaire » (Pierre).

Lorsque la mission est éloignée du domicile, les intérimaires sont logés le plus souvent près des chantiers et dans des logements collectifs. «Bon... On n'accepte pas une mission pour le logement. Ça va... on sait que c'est temporaire... Il faut faire le travail, c'est tout, et on rentre à la maison » (Enriko). 
Les conditions de travail sont jugées correctes. L'un d'eux estime néanmoins que des améliorations doivent encore être faites sur certains points. Certes, les agences d'intérim ont fait des efforts importants de sensibilisation aux risques d'accidents du travail. Les interviewés soulignent par exemple le fait que les agences font passer des tests sécurité à pratiquement tous les intérimaires qu'elles détachent. Des formations à la sécurité ont également été organisées ces dernières années, particulièrement dans le secteur du bâtiment où les taux d'accidents du travail sont deux fois plus importants que dans les autres secteurs (Belkacem, Montcharmont, 2012).

\section{Une pratique légale, contestée, mal contrôlée}

Les détachements de travailleurs au sein de l'UE et leur encadrement règlementaire alimentent un débat qui reprend de l'ampleur aujourd'hui. L'amélioration des règles européennes sur le sujet, et en particulier le contrôle des pratiques mises en œuvre, apparaît de plus en plus nécessaire, sinon pour tous les États-membres, en tout cas pour la Commission européenne et pour la France, voire pour l'Allemagne.

Le détachement transfrontalier sur intérim est bien sûr un cas très particulier de détachement et ne saurait représenter l'ensemble des situations de détachement. Il reste que ce cas très particulier est intéressant à étudier. Il parait bénéficier autant aux employeurs qu'aux salariés. Et pourtant, il n'est pas lui non plus à l'abri des abus. Mieux, il fait apparaitre que des abus peuvent être pratiqués en toute légalité. Nos sources d'information proviennent ici d'entretiens réalisés auprès des principaux acteurs du domaine : les agences d'intérim employeurs, les syndicats de salariés, les responsables lorrains de l'inspection du travail. Au-delà des quelques chiffres disponibles, la réalité du phénomène que l'on y saisit donne un éclairage étonnant.

\section{IV.1. Les responsables patronaux luxembourgeois : une activité légale de gestion de la main-d'œuvre frontalière}

Les responsables d'agences d'intérim luxembourgeoises soulignent que le détachement du Luxembourg vers la Lorraine est une pratique conforme aux réglementations nationale et européenne. «Aucun texte réglementaire n'interdit la liberté de circulation des travailleurs au sein de l'espace européen. » Mieux, ce serait « un système gagnant-gagnant pour les travailleurs qui touchent des salaires plus élevés, et pour les entreprises qui ont moins de charges ». Le prix de la prestation de service d'intérim de statut luxembourgeois est avantageux pour tous, l'entreprise utilisatrice et les intérimaires eux-mêmes. 
Mais en France, on le sait, les responsables patronaux de l'intérim soulignent au contraire la concurrence légale certes, mais déloyale, que leur font les sociétés d'intérim luxembourgeoises. Ce sont bien ici les différentiels de législations sociales et fiscales qui favoriseraient l'essor des sociétés d'intérim luxembourgeoises et l'intérim transfrontalier.

Pour développer cette activité de détachement, ces sociétés d'intérim luxembourgeoises ont recherché un fort ancrage territorial au cœur de l'espace transfrontalier. Depuis les années 1990, dans un contexte de développement important du travail frontalier, elles ont multiplié leurs localisations d'agences tout le long des frontières du Nord-Est (avec l'Allemagne) et du Sud du Luxembourg (avec la France) face aux anciens bastions industriels caractérisés par des taux de chômage élevés et donc par la présence d'une réserve abondante de main-d'œuvre (Belkacem et al., 2007). Ces intermédiaires de l'emploi drainent ainsi une grosse partie de la main-d'œuvre frontalière (Belkacem, Kratz, 2013). Pour le compte des entreprises utilisatrices, tout en contribuant à abaisser le coût du travail, elles remplissent les fonctions de gestion de la main-d'œuvre que cherchent classiquement à s'approprier les sociétés d'intérim : (i) drainer la main-d'œuvre disponible sur leur territoire, voire au-delà grâce aux réseaux qu'elles tissent jusqu’à l'international, sous la même enseigne ; (ii) sélectionner et recruter les candidats ; (iii) déléguer ou détacher des travailleurs sélectionnés sur les emplois sur lesquels ils sont les plus susceptibles de donner satisfaction ; (iv) conseiller les entreprises utilisatrices sur leurs meilleurs choix d'affectation de la main-d'œuvre disponible sur le marché.

L'ancrage territorial des agences d'intérim le long des frontières permet de traverser ces frontières plus aisément, pour élargir leurs réservoirs de candidats à l'intérim. Ces agences ont su développer des partenariats avec les intermédiaires de l'emploi de l'autre côté des frontières. Par exemple, des collaborations de sociétés luxembourgeoises avec Pôle emploi en France existent de longue date. Cela se traduit par des échanges réguliers d'informations sur des candidats potentiels et des offres de missions d'intérim. Des actions de communication sont régulièrement menées directement à destination des étudiants des écoles et des instituts universitaires de technologie (IUT) des villes frontalières (conférences, distributions de tracts de présentation, propositions de stage...). Des contacts réguliers existent avec d'autres agences du même groupe géographiquement décentralisées de l'autre côté des frontières qui facilitent l'organisation de " pools» élargis de main-d'œuvre pour la recherche de candidats. Les réseaux facilitent bien sûr l'optimisation des différentiels de législations sociales et fiscales en fonction des lieux de localisation des entreprises utilisatrices. 


\section{IV.2. Les difficultés des syndicalistes français pour la défense des travailleurs détachés}

Entre les syndicalistes et les travailleurs détachés, a fortiori détachés sur intérim, la distance est loin de se réduire. On le sait, même en interne, au plan national, la situation des intérimaires ne facilite jamais l'action syndicale (Belkacem et al., 2014a, 2014b). Les différences de statut entre intérimaires et personnels permanents ne sont pas faciles à gérer. Concernant les intérimaires de statut français et les détachés transfrontaliers, les syndicalistes notent des difficultés assez semblables finalement, mais sans doute amplifiées.

Le statut privilégié des intérimaires luxembourgeois ne les incite évidemment pas à s'adresser à un syndicat en cas de conflit. Bien entendu, ce statut ne leur épargne pas la peur classique de tout salarié précaire de sanctions ou de représailles de la part des employeurs s'ils approchent les syndicalistes. Les directions des entreprises utilisatrices ne facilitent pas les interventions syndicales, ne serait-ce qu'en termes d'accès aux sites de travail. Et même, dans le cas des chantiers de construction, les détachés sont regroupés et éloignés, pratiquement isolés des personnels permanents. Enfin et surtout, la parfaite légalité de la situation du détachement transfrontalier évacue bien des motifs d'action syndicale.

Outre les formes d'empêchement de la part des employeurs, d'autres barrières objectives à la représentation et la mobilisation de ces travailleurs précaires se manifestent fréquemment. Les travailleurs détachés sont recrutés pour des missions ponctuelles et de durées courtes. Ils sont perçus par les salariés des entreprises utilisatrices comme une main-d'œuvre temporaire et/ou fréquemment renouvelée. Les occasions pour établir des formes de socialisation collectives demeurent limitées. Les organisations syndicales priorisent leurs actions en visant tout naturellement le noyau dur des travailleurs. Lorsque l'entreprise utilisatrice est aux prises à de grosses difficultés qui menacent d'affecter l'emploi, la défense des permanents devient prioritaire, les détachés font figure de concurrents extérieurs. Les salariés dits "sous-traités » sont peu pris en compte, peu défendus. Un responsable de l'union départementale de Meurthe-et-Moselle de la CGT interrogé commente à propos du détachement de salariés étrangers dans son entreprise : «Dans l'entreprise - j'y suis depuis plus de trente ans -, dès le départ, la sous-traitance a commencé en 1986, on nous a expliqué que "c'est eux qui viennent piquer notre boulot !" (...) J'ai entendu des militants avoir des réflexions et dire: "Oui, ils viennent piquer notre travail !" Alors que le travail est là, et il y a une mise en concurrence des salariés. (...) Oui, pour moi, ça c'est tabou, le sujet du détachement. »

La situation n'est pas ou peu différente des problématiques de l'action collective déjà étudiées sur les missions d'intérim standard, sur statut français. Mais l'aspect transfrontalier met au premier plan la difficulté que 
rencontrent les syndicalistes pour bien mesurer toute la complexité, on peut même dire toute la sophistication des fraudes auxquelles donne lieu le détachement transfrontalier. Avec la bénédiction des utilisateurs, et ici peut-être plus qu'ailleurs, des travailleurs intérimaires eux-mêmes. Ici, c'est l'inspection du travail française qui possède les moyens d'en évaluer toute l'ampleur et surtout la sophistication des procédures peu légales sinon clairement frauduleuses.

\section{IV.3. L'inspection du travail : contrôler les détachements, une mission décisive mais des moyens limités}

Une responsable de la Direccte de Meurthe et Moselle nous confiait que le contrôle du travail en détachement constitue aujourd'hui « une priorité nationale », au même titre que "la négociation administrée » (c'est-àdire le contrôle du bon fonctionnement des institutions représentatives du personnel), ou la sécurité au travail. Les inspecteurs du travail sont là pour « veiller au respect du noyau dur. Le noyau dur, c'est la durée du travail, c'est le salaire, c'est l'hébergement, et la sécurité/santé des gens. » Mais la difficulté majeure du contrôle est bien expliquée par le directeur adjoint responsable de l'unité régionale de lutte contre le travail illégal (Uracti ${ }^{9}$ ) Lorraine : "Ce qui n’est pas déclaré, on ne le connait pas par définition. On va les connaitre, par hasard, on en trouve! Mais par définition, on les trouve moins facilement que ceux qui sont déclarés. Et ceux qui sont déclarés, on va les contrôler pour vérifier que la réalité est conforme à ce qui est marqué dans la déclaration. » Paradoxalement, les entreprises qui semblent être le plus dans l'illégalité sont donc moins soumises à des contrôles.

Les fraudes peuvent être très complexes. Ce responsable de l'Uracti observait que le protocole de contrôle que doivent suivre les inspecteurs du travail induit systématiquement la vérification de l'existence réelle des entreprises. Il faut vérifier en particulier « que l'entreprise a une existence réelle au Luxembourg. Il y a quand même des entreprises boîtes aux lettres. Ce sont nos collègues au Luxembourg qui le disent. Pour qu'il y ait détachement, il faut que l'entreprise ait une activité réelle dans le pays d'origine. Le problème se pose aussi en Pologne ou en Slovaquie. On pourrait avoir des entreprises fictives en Pologne ou en Slovaquie, surtout que là, c'est un peu plus difficile à vérifier! Au Luxembourg, on a quand même des facilités avec l'inspection du travail luxembourgeoise et on arrive à peu près à voir ce qui se passe. Premier problème : il faut que l'entreprise ait une activité réelle. »

Nos interlocuteurs soulignaient que ces pratiques du détachement tendent à être de plus en plus organisées, structurées et déguisées. Les abus sont nombreux, et difficilement identifiables.

9. L'Uracti est un des services de l'inspection du travail chargé plus spécifiquement du contrôle du détachement de travailleurs. 
Le lieu de signature du contrat de mission par l'intérimaire est décisif : d'un côté à l'autre de la frontière, les règles changent. En implantant des agences d'intérim de part et d'autre des frontières, « une société mère à un endroit, une agence localisée à un autre endroit», les montages d'opérations peuvent être complexes et par conséquent difficilement identifiables. «On a quelques entreprises luxembourgeoises qui ont des agences en France. Imaginons qu'une entreprise luxembourgeoise basée sur Esch-sur-Alzette veuille embaucher un salarié français. Pour le salarié français, ce ne sera pas trop compliqué de passer la frontière. Il fera le plein d'essence. Mais s’il habite à Lunéville ou à Épinal ou plus loin, là, il y a beaucoup moins de chances qu'il soit allé au Luxembourg pour signer son contrat. Une entreprise luxembourgeoise peut très bien signer un contrat en France mais là, le contrat est soumis à la loi française et elle ne peut pas le détacher, puisqu'il a été détaché en France! C'est la deuxième fraude, assez difficile à repérer quand même, mais qui peut exister » (responsable de l'Uracti Lorraine).

Les fraudes les plus importantes sont la non-déclaration de travailleurs détachés. Les discours des responsables de l'inspection du travail soulignent à la fois l'importance de ce problème et la relative impuissance de l'inspection du travail qui ne dispose ni des informations nécessaires, ni des moyens humains pour repérer les entreprises qui recourent à des salariés détachés sans en informer l'inspection du travail. Les contrôles de l'inspection du travail seraient en forte progression ces dernières années, mais ne donneraient lieu qu'à très peu de sanctions. Par ailleurs, seuls $3 \%$ des constats d'infractions faits par les inspecteurs du travail seraient relevés par procès-verbal ou, pour le dire autrement, « $97 \%$ des constats donnent lieu à des rappels à la loi, des observations, des explications de texte. (...) $3 \%$ des infractions sont relevées par PV. $97 \%$ ce sont du conseil, des rappels à la loi. » 500 contrôles auraient été réalisés en 2015 dans des établissements et sur des chantiers en Lorraine. En tout, 86 procès-verbaux auraient été dressés auxquels il faut ajouter des « signalements au Parquet». 200 contrôles ont été effectués dans le milieu du bâtiment, pour seulement 26 procès-verbaux. «Quatre ans après les transmissions [des procèsverbaux aux Parquets], un tiers seulement des procédures suivies ont donné lieu à poursuites ${ }^{10}$. »

\section{Conclusion}

Être détaché peut permettre d'obtenir un meilleur salaire que dans son pays d'origine, d'obtenir un emploi pour sortir du chômage, d'avoir une opportunité de travailler temporairement à l'étranger. Pour l'entreprise, les avantages peuvent être l'embauche d'une main-d'œuvre à plus faible

10. Rapport annuel de la Cour des comptes, février 2016, p. 382. 
coût, le recrutement de personnels étrangers sur des métiers en tension ou encore de faciliter les mobilités intra-groupes.

Par ses caractéristiques géographiques et les liens historiques, culturels, économiques nouant les entités la constituant, la Grande Région Sarre-Lorraine-Luxembourg rassemble sur son territoire une proportion importante des travailleurs détachés européens, dont une large part de la main-d'œuvre transfrontalière intra-européenne, fréquemment sur intérim. Les kilomètres à parcourir et les difficultés socioculturelles pour devenir un travailleur détaché y sont bien moindres. Les témoignages recueillis de quelques salariés détachés dans la Grande Région tendraient même à montrer que l'expérience est presque banale.

Les différentiels de législations et de dynamiques économiques de part et d'autre des frontières paraissent constituer les ressorts essentiels du développement de ces pratiques de détachement. Les entreprises de travail temporaire luxembourgeoises et les entreprises utilisatrices localisées de l'autre côté des frontières (les entreprises françaises dans le cas de cette étude) exploitent naturellement ces différences de législation pour en tirer des avantages financiers.

Entre le Luxembourg et la France, la situation des intérimaires détachés est complètement à rebours de ce qui est souvent médiatisé, puisque c'est la France qui est le pays d'accueil et le régime français qui est moins favorable aux salariés que le régime luxembourgeois. Mais on l'a montré, il reste une difficulté majeure : le contrôle des pratiques illicites. Le discours que nous a tenu le secrétaire général de l'union lorraine de la fédération française du bâtiment est particulièrement explicite. "Le détachement est légal. Nous, ça y est, on a trouvé la formule maintenant. C'est "la fraude massive au détachement", pour essayer de mettre des mots qui vont bien. Le détachement, s’il est appliqué dans les règles, si le salarié est détaché, il y a uniquement le différentiel de charges. Si le salaire minimum, conventionnel est respecté, si la durée du travail est respectée, s’ils sont logés dans de bonnes conditions, il n'y a que le différentiel de charges. (...) Il y a une certaine concurrence, qui est légale. Mais, le problème c'est que ça n'est jamais appliqué comme cela. »

Nos résultats sont partiels, fragiles. Ils invitent pourtant à prolonger cette recherche sur au moins deux axes de questionnement, encore trop peu traités : d'une part le profil socioprofessionnel et les motivations des travailleurs détachés ; d'autre part les fondements économiques des relations qui se nouent entre les entreprises de travail temporaire luxembourgeoises et les entreprises utilisatrices localisées au-delà des frontières de cet État.

Enfin, nous n'avons traité que des utilisateurs localisés en France. On peut imaginer que les localisations d'utilisateurs en Allemagne, en Belgique, voire aux Pays-Bas soulèvent les mêmes questions. 


\section{Références bibliographiques}

Belkacem R., Bennoui M., Rouyer R. (2007), Le rôle du travail intérimaire dans la régulation du marché du travail au Grand-Duché de Luxembourg, Rapport final, Activités prévues dans le programme EURES 2006-2007.

Belkacem R., Kornig C., Michon F., Montcharmont L., Nosbonne C., Scalvinoni B. (2016), Les pratiques de détachements de travailleurs dans l'Union européenne : importance, forme et enjeux. Le cas de la grande région SarreLor-Lux, Agence d'Objectifs de l'Ires, étude $\mathrm{n}^{\circ} 2014-03$ réalisée pour Force ouvrière, Rapport final, décembre, https://goo.gl/ATXCZD.

Belkacem R., Kornig C., Michon F., Nosbonne C. (2014a), L'intérim : évolutions et syndicalisation, Agence d'objectifs de l'Ires, étude $n^{\circ}$ 2010-05 réalisée pour la CGT-Force ouvrière, janvier, http://pmb.cereq.fr/doc_num. php?explnum_id=1151.

Belkacem R., Kornig C., Michon F., Nosbonne C. (2014b), « Mobiliser, défendre les intérimaires. Les difficultés de l'action syndicale ", La Revue de l'IRES, $n^{\circ} 83$, p. 3-28, https://goo.gl/mrEwPj.

Belkacem R., Kratz R. (2013), « Le rôle des intermédiaires de l'emploi dans les processus d'ajustement entre la main-d'œuvre et les emplois disponibles en contexte transfrontalier », in Belkacem R., Pigeron-Piroth I. (dir.), Le travail frontalier au sein de la Grande Région Saar-Lor-Lux. Pratiques, enjeux et perspectives, Nancy, PUN, p. 433-448.

Belkacem R., Montcharmont L. (2012), « Analyse des conditions de travail des travailleurs intérimaires : Comment expliquer la vulnérabilité des intérimaires aux accidents du travail ? ", PISTES. Perspectives interdisciplinaires sur le travail et la santé, vol. 14, n 2, https://doi.org/10.4000/pistes.2543.

Belkacem R., Pigeron-Piroth I. (2011), « Travail frontalier et développement transfrontalier au sein de la Grande Région Saar-Lor-Lux », Géo-Regards, n 4, p. 13-28, https://goo.gl/Pp6Tqh.

Belkacem R., Pigeron-Piroth I. (2012), « Les travailleurs frontaliers au sein de la Grande Région Saar-Lor-Lux », in Belkacem R., Pigeron-Piroth I. (dir.), Le travail frontalier au sein de la Grande Région Saar-Lor-Lux. Pratiques, enjeux et perspectives, Nancy, PUN, p. 25-46.

DGT (2016), Analyse des déclarations de détachement des entreprises prestataires de services en France en 2015, Direction générale du travail, Ministère du Travail, de l'Emploi, de la Formation professionnelle et du Dialogue social, septembre, https://goo.gl/fiem4H.

Direccte (2015), PSI en Région Lorraine en 2013 et 2014, Région Lorraine, Documents internes.

Drees (2017), La protection sociale en France et en Europe en 2015 - résultats des comptes de la protection sociale, édition 2017, Direction de la recherche, des études, de l'évaluation et des statistiques (Drees), ministère des Solidarités et de la Santé, https://goo.gl/vAoGT3.

Eurostat (2017), "Statistiques sur la protection sociale », in Eurostat Statistics Explained, juin, http://ec.europa.eu/eurostat/statistics-explained/index.php/ Social_protection_statistics/fr.

European Commission (2016), Posting of Workers - Report on A1 portable documents issued in 2015, Directorate-General for Employment, Social Affairs and Inclusion, Unit D/2, European Commission, Brussels. 
Insee (2015), « Une comparaison du coût de la main-d'œuvre en Europe : quelle évolution depuis la crise ? ", in Dossier, Insee références, Statistiques publiques, édition 2015, https://www.insee.fr/fr/statistiques/1906513?sommai re $=1906539$.

Kornig C. (2007), "Choisir l'intérim : sous quelles conditions ? », Économie et Société, Cahiers de Socio-économie du travail, $\mathrm{n}^{\circ} 29$, décembre, p. 19591977, https://halshs.archives-ouvertes.fr/halshs-00436955/document.

Math A. (2006), « Les travailleurs détachés dans le cadre de la sous-traitance internationale », Migration Société, vol. 18, n 107, p. 65-83.

Statec (2012), Regards sur le travail intérimaire, $\mathrm{n}^{\circ} 3$, Luxembourg, http://www. statistiques.public.lu/fr/publications/series/regards/2012/03-12-interimaire/ index.html.

Statec (2014), Regards sur le coût de la main-d'oeuvre au Luxembourg et en Europe, $\mathrm{n}^{\circ} 22$, Luxembourg, http://statistiques.public.lu/fr/publications/series/ regards/2014/22-14-cout-main-doeuvre/index.html.

Thoemmes J. (2014), « Le travail détaché. Le cas des salariés portugais du secteur de la construction en France ", Les Mondes du travail, n 14, p. 39-55, https://hal.archives-ouvertes.fr/hal-01088250. 